\title{
Invasive fungal infection in patients with hematologic disorders in a Brazilian tertiary care hospital
}

\author{
Milton Camplesi Junior ${ }^{[1]}$, Hildene Meneses Silva ${ }^{[2]}$, Adriano Moraes Arantes ${ }^{[3]}$, \\ Carolina Rodrigues Costa ${ }^{[2]}$, Fábio Silvestre Ataides ${ }^{[2]}$, Thaisa Cristina Silva ${ }^{[2],}$ \\ Maysa de Paula Costa dos Reis ${ }^{[2]}$ and Maria do Rosário Rodrigues Silva ${ }^{[2]}$
}

\author{
[1]. Departamento de Biomedicina, Universidade Paulista, Goiânia, GO, Brasil. \\ [2]. Departamento de Microbiologia, Imunologia, Parasitologia e Patologia, Instituto de Patologia Tropical, \\ Universidade Federal de Goiás, Goiânia, GO, Brasil. [3]. Hospital Araújo Jorge, Goiânia, GO, Brasil.
}

\begin{abstract}
Introduction: Invasive fungal infections (IFIs) are an important complication in immunocompromised individuals, particularly neutropenic patients with hematological malignancies. In this study, we aimed to verify the epidemiology and diagnosis of IFIs in patients with hematologic problems at a tertiary hospital in Goiânia-GO, Brazil. Methods: Data from 117 patients, involving 19 cases of IFIs, were collected. The collected data included diagnosis methods, demographics, clinical characteristics, and in vitro susceptibility to different antifungal agents. Among the 19 cases, 12 were classified as proven IFI and 7 as probable invasive aspergillosis with detection of galactomannan in blood and presence of lung infiltrates in radiographic images. Logistic regression analysis showed that the proven and probable IFIs were associated with increased risk of death. Statistical analysis demonstrated that age, sex, and underlying disease were not independently associated with risk of death in IFI patients. Results: Most bloodstream isolates of Candida spp. exhibited low minimum inhibitory concentrations (MICs) to all antifungal agents tested. Voriconazole and amphotericin had the lowest MICs for Aspergillus spp. and Fusarium spp., but Fusarium spp. showed the least susceptibility to all antifungals tested. Amphotericin B, fluconazole, and itraconazole were found to be inactive in vitro against Acremonium kiliense; but this fungus was sensitive to voriconazole. Conclusions: Considering the high number of IFI cases, with crude mortality rate of $6 \%$, we could conclude that IFIs remain a common infection in patients with hematological malignancies and underdiagnosed ante mortem. Thus, IFIs should be monitored closely.
\end{abstract}

Keywords: Invasive fungal infections. Hematologic disease. In vitro susceptibility.

\section{INTRODUCTION}

Invasive fungal infections (IFIs) are an important complication in immunocompromised individuals, particularly in neutropenic patients with hematological malignancies ${ }^{1}$. Over the last decades, this group of patients has presented severe infections, which has been the cause of high mortality rate ${ }^{2,3}$.

Invasive aspergillosis (IA) and invasive candidiasis are the main invasive fungal diseases associated with bloodstream infections. Although invasive yeasts, such as Candida spp., and molds, such as Aspergillus spp., are the predominant pathogens of IFIs, other uncommon and difficult-to-treat molds, such as Mucorales, Fusarium spp., and phaeohyphomycetes, have emerged in patients with hematological malignancies ${ }^{4,5}$. The clinical presentation of IFIs is nonspecific, and the diagnostic criteria are poorly defined in critically ill populations, rendering

Corresponding author: Dra. Carolina Rodrigues Costa.

e-mail: carolrc80@yahoo.com.br

Received 10 June 2016

Accepted 7 February 2017 the diagnosis of the disease challenging. In this study, we aimed to verify the epidemiology and diagnosis of IFIs in patients with hematologic problems at a tertiary hospital in Goiânia-GO, Brazil.

\section{METHODS}

\section{Patients and study setting}

This study assessed the prevalence (16.2\%) of IFIs among 117 patients with hematologic malignancies in Hospital Araújo Jorge/Associação de Combate ao Câncer em Goiás (ACCG) of Goiânia-GO from December 2009 to July 2011. The setting was a 170-bed tertiary care hospital with medical and surgical wards, intensive care units, and an emergency department. The following variables were recorded and analyzed: sex, age, and underlying disease. This study was approved by the Ethics Committee of ACCG. Hospital prophylactic protocols included antifungal drugs such as fluconazole or itraconazole for every patient.

The only cases included in the data analysis were those classified as proven or probable according to the European Organization for Research and Treatment of Cancer/Mycosis Study Group ${ }^{6}$ (EORTC). IFIs were defined as proven when 
cultures from blood were positive for fungi (at least twice at different times). IFIs were defined as probable IA when new lung infiltrates were evidenced on high-resolution chest computed tomography scans and galactomannan (GM) was detected in serum with the Platelia Aspergillus enzyme immunoassay.

Blood cultures were performed to detect fungal species by using a biphasic brain-heart infusion (BHI) medium. Five milliliters of blood were inoculated into the culture bottles and incubated in an upright position for 30 days at $25^{\circ} \mathrm{C}$. Cultures were examined daily and subcultured onto Sabouraud dextrose agar slant medium for identification. The isolated filamentous fungi were identified with standard morphological characteristics of their conidiophores and conidia. For species identification, a slide culture method was used (microculture) in potato agar and the culture was incubated at $25^{\circ} \mathrm{C}$ for 5 days. Confirmation of fungi as etiologic agent was accomplished by reproducible growth from the blood samples at different times by repeated culture attempts. Candida isolates were identified by microscopic morphology on cornmeal tween 80 agar and by biochemical methods using the API 32C AUX system (Biomerieux, Marcy l'Etoile, France).

Antifungal susceptibility tests to four antifungal agents, namely amphotericin $B$, itraconazole, voriconazole, and fluconazole, were performed using the broth microdilution assay. These tests were carried out according to the Clinical and Laboratory Standards Institute (formerly the National Committee for Clinical Laboratory Standards) M27-A3, M38-A2, and M27-S4 documents ${ }^{7-9}$.

Serum GM antigen levels were measured weekly using enzyme linked immunosorbent assay (ELISA) with the Platelia Aspergillus test (Bio-Rad, Paris, France). GM testing was conducted in accordance with the manufacturer's recommendation for testing serum samples. A cutoff optical density index of $0.5 \mathrm{ng} / \mathrm{mL}$ or higher was considered positive for GM in serum in two sequential samples ${ }^{1,10}$.

\section{Statistical analysis}

All statistical analyses were performed with SPSS ${ }^{\circledR}$ software (version 16 for Windows). Univariate analysis, which was determined using chi-square $\left(\chi^{2}\right)$ analysis, identified variables predicting mortality. The following variables were recorded and analyzed: sex, age, and underlying disease. The significance level was set at 5\%. Sensitivity and specificity were reported for GM testing.

\section{RESULTS}

The mean age of the 117 patients was 35.7 years, with a range of 21 to 60 years. Of these, $52.1 \%$ were male and $24.7 \%$ had acute myeloid leukemia (Table 1). Ninety percent of the patients were severely neutropenic at the time of symptom

\section{TABLE 1}

Analysis of characteristics and risk factors of 117 patients with hematopoietic stem cell transplant for invasive fungal infections (proven/probable).

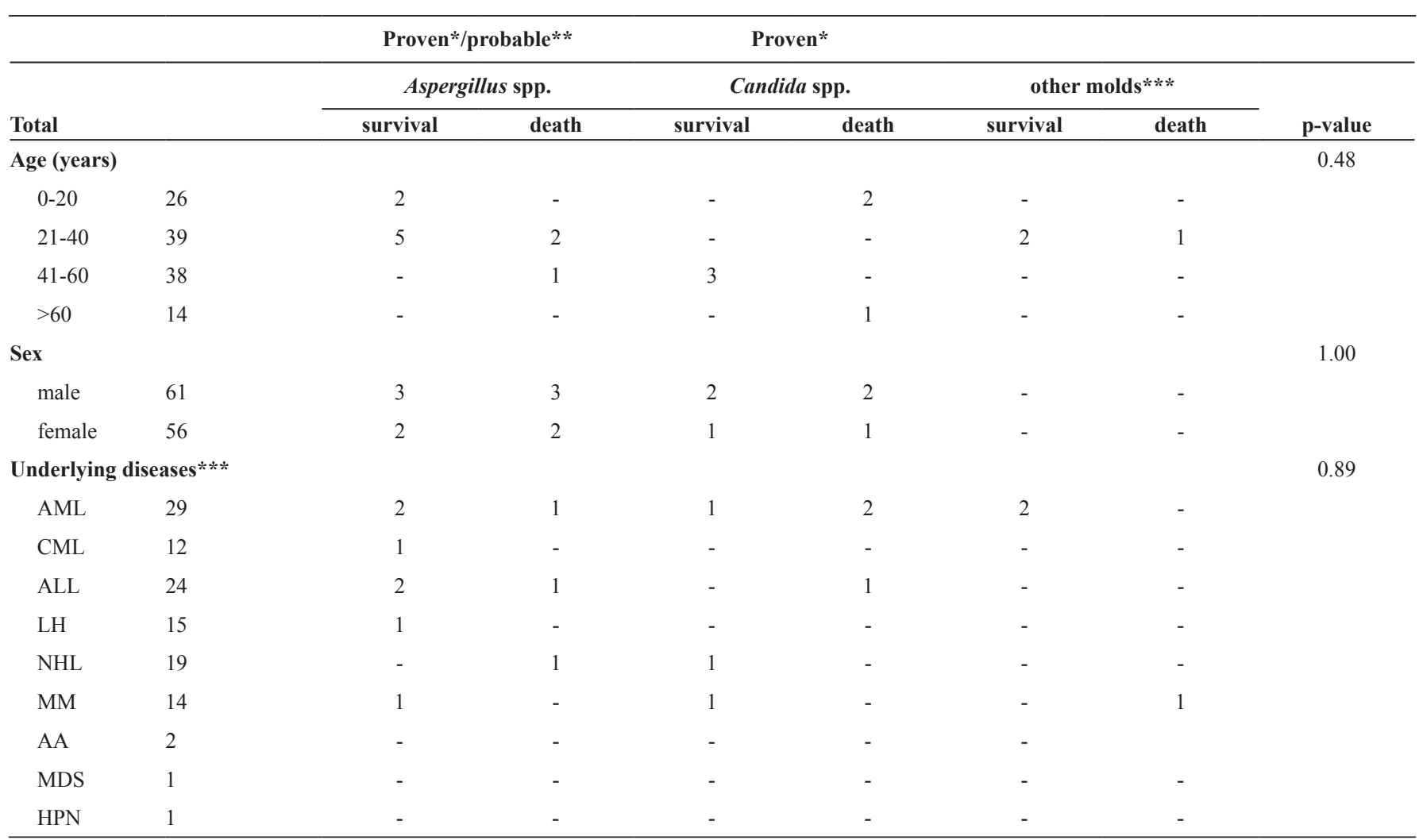

AML: acute myeloid leukemia; CML: chronic myeloid leukemia; ALL: acute lymphoid leukemia; LH: Hodgkin's lymphoma; NHL: non-Hodgkin lymphoma; MM: multiple myeloma; AA: aplastic anemia; MDS: myelodysplastic syndrome; HPN: paroxysmal nocturnal hemoglobinuria. *Proven: positive culture for fungi. **Probable: clinical illness consistent with IFI with supporting radiographic image and detection of galactomannan in serum. *** Other molds: Fusarium spp. (2 cases), Acremonium kiliense (1 case). 
onset. IFIs were identified as proven in $12(10.2 \%)$ patients, defined by growth of Aspergillus spp., Candida spp., Fusarium spp., and Acremonium kiliense on BHI medium. Among the culture-documented infections, Aspergillus fumigatus accounted for two samples, A. flavus for one sample, and C. albicans was responsible for the majority ( 3 cases) of yeast invasive infections, followed by C. parapsilosis and C. tropicalis (Table 2). Probable IA was found in seven patients (6\%), with detection of GM in blood and presence of lung infiltrates in radiographic images (Figure 1). All patients diagnosed with IFIs had used fluconazole as the prophylactic agent. The median time of IFI diagnosis was 45 days (range 7-84 days) since hospitalization. Out of the 10 patients with proven and probable IA, nine (90\%) exhibited at least one symptom that was suggestive of pulmonary disease and one had disseminated infection. Disseminated infection with Fusarium spp. occurred from skin as a portal of entry, whereas $A$. kiliense fungemia had involvement of the lungs.

The overall mortality rate for hematologic disorders with proven/probable IFI was $6 \%(7 / 117)$. In the analysis of underlying diseases alone or together, the $p$ values were $>0.05$. Demographic characteristics, underlying disease, and outcomes of patients associated with IFIs found in this study are outlined in Table 1.

As shown in Table 2, most Candida bloodstream isolates exhibited low minimum inhibitory concentrations (MICs) to all antifungal agents tested. Amphotericin B had the lowest MICs ranging from 0.125 to $0.5 \mu \mathrm{g} / \mathrm{mL}$ for all species, while resistance to all azoles tested was observed in one strain of $C$. albicans. The MICs for filamentous fungi ranged markedly among antifungal agents and organisms. Voriconazole and amphotericin B had the lowest MICs against Aspergillus spp. However, Fusarium spp. were the least susceptible against all antifungals tested, inhibited

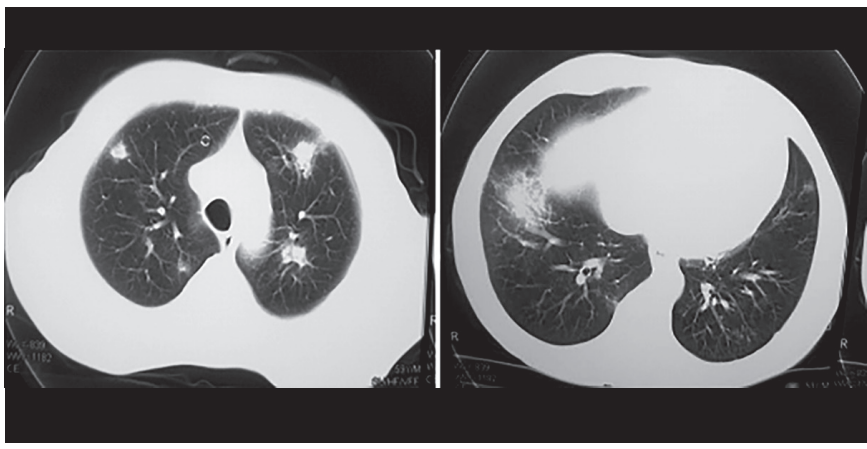

FIGURE 1 - Chest computed tomography: pulmonary infiltrates scattered throughout the parenchyma of both lungs evidenced in probable cases of invasive aspergillosis.

only at high concentrations of itraconazole and fluconazole. Amphotericin B, fluconazole, and itraconazole were found to be inactive in vitro against $A$. kiliense, but $A$. kiliense was sensitive to voriconazole.

\section{Statistical analysis and diagnostic performance of serum galactomannan assay}

Statistical analysis revealed that age, sex, and underlying disease were not independently associated with risk for IFI. Hematologic diseases were associated with increased risk for IFI by molds $(p<0.01)$. Seven $(6 \%)$ died among the IFI patients, and analysis identified the association of IFIs with increased risk of death $(p<0.01)$ in these patients.

The performance of the GM test was evaluated with proven and probable IA. At the index cutoff value of $\geq 0.5 \mathrm{ng} / \mathrm{mL}$, the ELISA tests had a sensitivity of $90 \%$ ( 9 of 10) and a specificity of $85.8 \%(94 / 107)$.

TABLE 2

Antifungal susceptibility of bloodstream isolates from patients with hematologic malignances.

\begin{tabular}{|c|c|c|c|c|}
\hline \multirow[b]{2}{*}{ Species (n) } & \multicolumn{4}{|c|}{ MIC $(\mu \mathrm{g} / \mathrm{mL})$} \\
\hline & Fluconazole & Voriconazole & Amphotericin B & Itraconazole \\
\hline Candida parapsilosis (2) & 0.125 & $<0.003$ & 0.125 & 0.5 \\
\hline \multirow[t]{2}{*}{ Candida albicans (3) } & 0.5 & $<0.003$ & 0.25 & $<0.003$ \\
\hline & 0.5 & 0.19 & 0.5 & 0.125 \\
\hline Candida tropicalis (1) & 8 & 0.006 & 0.5 & $>0.003$ \\
\hline \multirow[t]{2}{*}{ Aspegillus fumigatus (2) } & 16 & 0.25 & 2 & 0.12 \\
\hline & 32 & 0.5 & 4 & $>16$ \\
\hline A spegillus flavus (1) & $>64$ & 0.5 & 2 & 0.25 \\
\hline
\end{tabular}

MIC: minimum inhibitory concentration. 


\section{DIscussion}

Invasive mycoses are life-threatening opportunistic infections and have emerged as a major cause of morbidity and mortality in critically ill patients ${ }^{11}$. This study is the first to verify the prevalence of IFIs in hematologic malignancy patients in Goias State (Midwest of Brazil). In our survey, aspergillosis and candidemia were the major IFIs detected in patients with hematologic malignances, but other infections, such as fusariosis and $A$. kiliense fungemia with involvement of the lungs, were observed.This study focused on the epidemiology of fungal diseases, their considerable morbidity in patients with hematologic malignances, and low sensitivity of the culture methods for the detection of Aspergillus infections. We observed IFIs in $19(16.2 \%)$ patients with hematologic disorders, with molds present in 13 and yeasts in 6 patients. An increased incidence of IFIs that present hematologic problems was reported by some researchers ${ }^{12,13}$. Singh and Paterson ${ }^{14}$ verified that IA is the most frequent IFI; it can occur in $8-15 \%$ of patients with hematologic disease. In the present study, Aspergillus infections were considered as proven/probable in $10(8.5 \%)$ of 117 hematologic problem patients. The diagnosis of proven IA infection represented a problem in this study. IA diagnosis is a challenge because clinical and radiological signs are nonspecific, and tissue biopsies are invasive and not always a possible means of diagnosis ${ }^{10}$. Among the 10 patients with suspected aspergillosis, infection was proven in only $30 \%$ of cases by blood culture and probable according to EORTC criteria in $70 \%$. In these patients, we detected GM antigenemia and lung infiltrates using standard chest radiography and high-resolution computerized tomography.

The impact of early diagnosis leading to appropriate therapy may result in improved prognosis of patients. In this way, the detection of GM, a component of cell wall of Aspergillus sp. in body fluids hematogenously released during hyphal growth, becomes a useful aid in IA diagnosis ${ }^{10,15}$. The assay has gained widespread acceptance as a sensitive method to undertake prospective surveillance in risk population ${ }^{16}$. The sensitivity and specificity of GM testing found in our study (with at least two consecutive positive samples) were $90 \%$ (9 out of 10) and $85.8 \%$ (94/107), respectively, when IA was defined using clinical characteristics and radiological findings suggestive of aspergillosis and even taking into account the positive culture. The Platelia Aspergillus GM EIA was a disappointment in one case where the culture was positive. Marr et al. ${ }^{17}$ suggested that antifungal therapy decreases the sensitivity of the Aspergillus GM enzyme immunoassay on serum samples. Antifungal prophylaxis using fluconazole was performed on all our patients. A high specificity of $99.6 \%$ was found by Pinel et al. ${ }^{18}$ with positive GM antigenemia in 748 of 751 patients. Several prior studies demonstrated IA as a predictor of poor outcomes ${ }^{19,20}$. Among patients classified as proven/probable IA, we also observed a significant predictor of death by logistic regression analysis ( $p<0.01$ ). This fungal infection occurs in $0.08-15 \%$ of patients with hematologic malignancies and presents mortality rates up to $80 \%{ }^{14,21}$. Then, we suggest that the Platelia Aspergillus GM EIA in patient serum could be useful as a screening tool for the identification of patients at a high risk of developing IA, thus reducing the percentage of deaths.
The real incidence of resistance in Aspergillus sp. is relatively unknown. However, azole resistance has been increasingly reported in many other countries since the detection of first azole-resistant isolate in 1997 in the $\mathrm{UK}^{22,23}$. In the Netherlands, azole resistance, particularly itraconazole, has an overall prevalence of $5.3 \%$, which ranges from 1.8 to $12.8 \%{ }^{24}$. Similarly, itraconazole resistance has been reported in other countries, with rates ranging from 1.7 to $6 \%{ }^{25}$. We also verified in our results that one isolate was resistant to itraconazole (Table 2). The in vitro and in vivo correlations of azole resistance are widely documented, with a clear association of resistant A. fumigatus strain isolation and lack of patient response to therapy ${ }^{24}$.

Although non-Aspergillus molds are rarely found with three cases, they remain a problem in patients with hematologic disease. The rare molds (2.5\%) diagnosed in our population (Fusarium and Acremonium from blood) were identified by culture, underlining the importance of cultivating every probable fungal material to achieve a diagnosis. Management of IFIs is a challenge among emerging fungal pathogens and it generally shows poor response to many antifungals ${ }^{26,27}$. The capability of reference method for antifungal susceptibility testing to detect emerging resistance patterns provides useful information to optimize the effectiveness of antifungal therapy. In our study, the investigation of the in vitro efficacy of several antifungals against different isolates showed that voriconazole and amphotericin B presented low MIC against Fusarium spp. In larger studies of isolates from systemic disease, voriconazole and amphotericin B have been shown to be effective against Fusarium spp. and Aspergillus spp. ${ }^{28,29}$. Santhanan et al. ${ }^{30}$ verified that Fusarium spp. show low susceptibility to itraconazole and voriconazole.

Little information is available about the susceptibility of Acremonium spp. to antifungals, but they are characteristically resistant to anti-Candida agents, such as fluconazole and flucytosine ${ }^{31}$. Although breakpoint data for those organisms are lacking, MICs of amphotericin B are commonly elevated, which suggests the poor activity of this drug. A. kiliense seems to be less susceptible to amphotericin B, fluconazole, and itraconazole as supported by our results. Low MICs to voriconazole were found, and success was obtained after clinical treatment with this drug. Finally, voriconazole may be the best therapeutic alternative.

Although yeast infections appear to be decreasing and shift to non-albicans Candida spp., probably because of azoles as prophylactic agents, they remain a significant problem in hematologic malignances with poor outcome in candidemia cases $^{11,32}$. The ratio of $C$. albicans to non-albicans species found in our study was 1:1 (Table 2). All patients were exposed to fluconazole or itraconazole as a prophylactic treatment. Therefore, the obtained results may be due to local epidemiology impact because C. albicans, C. parapsilosis, and C. tropicalis are the most frequently isolated species in Latin America. Moreover, according to several researchers, C. parapsilosis is often associated with the presence of intravascular catheters and is not influenced by exposure to fluconazole or other antifungal agents $^{33-35}$. In our study, we found six cases of candidiasis 
and three of them were lethal. Bergamasco et al. ${ }^{36}$ found that C. albicans is the most frequent species, followed by $C$. tropicalis and C. parapsilosis in hematologic malignancy patients. They also showed that candidemia is associated with mortality. Our susceptibility data showed one C. albicans isolate only, with reduced susceptibility to all azoles (Table 2). Cross-resistance between fluconazole and voriconazole has been frequently reported in many species of Candida ${ }^{37,38}$.

Considering that the crude mortality rate of IFIs was very high (6\%) and early therapy may lead to improved prognosis, we suggest that efforts should be expended to reach an early fungal diagnosis in populations with hematologic disease. Similarly, in vitro susceptibility can be extremely useful in adjusting therapy against these infections. In summary, IFIs remain a common infection in patients with hematological malignancies being frequently disseminated and underdiagnosed ante mortem. Thus, IFIs should be monitored closely.

\section{Conflicts of interest}

The authors declare that there is no conflict of interest.

\section{REFERENCES}

1. Tan BH, Low JGH, Nidhi L, Chlebicka NL, Kurup A, Foong Koon Cheah FK, et al. Galactomannan-guided preemptive vs. empirical antifungals in the persistently febrile neutropenic patient: a prospective randomized study. Inter J Infect Dis. 2011;15(5):350-56.

2. Alangaden GJ. Nosocomial fungal infections: epidemiology, infection control and prevention. Infect Dis N Am. 2011;25(1):20125.

3. Garcia-Vidal C, Viasus D, Carratala J. Pathogenesis of invasive fungal infections. Curr Opin Infect Dis. 2013;26(5):270-76.

4. Erjavec Z, Kluin-Nelemans H, Verweij PE. Trends in invasive fungal infections, with emphasis on invasive Aspergillosis. Clin Microbiol Infect Dis. 2009;15(7):625-33.

5. Wanke B, Lazéra MS, Nucci M. Fungal Infections in the Immunocompromised Host. Mem Inst Oswaldo Cruz. 2000;95 (Suppl I):153-58.

6. Ascioglu S, Rex JH, de Pauw B, Bennett JE, Crokaert F, Denning DW, Invasive Fungal Infections Cooperative Group of the European Organization for Research and Treatment of Cancer; Mycoses Study Group of the National Institute of Allergy and Infectious Diseases, et al. Defining opportunistic invasive fungal infections in immunocompromised patients with cancer and hematopoietic stem cell transplants: an international consensus. Clin Infect Dis. 2002; 34(1):7-14.

7. Clinical and Laboratory Standards Institute. Reference method for broth dilution antifungal susceptibility testing of yeast. CLSI document M27-A3. Clinical and Laboratory Standards Institute, Wayne, $\mathrm{Pa}, 2008 \mathrm{a}$.

8. Clinical and Laboratory Standards Institute. Reference method for broth dilution antifungal susceptibility testing of filamentous fungi. CLSI document M38-A2. Clinical and Laboratory Standards Institute, Second Edition Wayne, Pa, 2008b.

9. Clinical and Laboratory Standards Institute. Reference method for broth dilution antifungal susceptibility testing of yeast. CLSI document M27-S4. Clinical and Laboratory Standards Institute, Wayne, $\mathrm{Pa}, 2012$.
10. Pfeiffer CD, Fine JP, Safdar N. Diagnosis of invasive aspergillosis using a galactomannan assay: a meta-analysis. Clin Infect Dis. 2006; 42(10):1417-27.

11. Nosari AM, Caira M, Pioltelli ML, Fanci R, Bonini A, Cattaneo $\mathrm{C}$, et al. Hema e-chart registry of invasive fungal infection in haematological patients: improved outcome in recent years in mould infections. Clin Microbiol Infect. 2013;9(8):757-62.

12. Baddley JW, Andes DR, Marr KA, Kontoyiannis DP, Alexander BD, Kauffman CA, et al. Factors associated with mortality in transplant patients with invasive aspergillosis. Clin Infect Dis. 2010;50(5):1559-67.

13. Nucci M, Garnica M, Gloria AB, Lehugeur DS, Dias VCH, Palma $\mathrm{LC}$, et al. Invasive fungal diseases in haematopoietic cell transplant reciepients and in patients with acute myeloid leukaemia or myelodysplasia in Brazil. Clin Microbiol Infect. 2013;19(8):745-51.

14. Singh N, Paterson DL. Aspergillus infections in transplant recipients. Clin Microbiol Rev. 2005;18(1):44-69.

15. Husain S, Paterson DL, Studer SM, Crespo M, Pilewski J, Durkin $\mathrm{M}$, et al. Aspergillus galactomannan antigen in the bronchoalveolar lavage fluid for the diagnosis of invasive aspergillosis in lung transplant recipients. Transplantation. 2007; 83(10):1330-36.

16. Marr KA, Carter RA, Boeckh M, Martin P, Corey L. Invasive aspergillosis in allogeneic stem cell transplant recipients: changes in epidemiology and risk factors. Blood. 2002;100(13):4358-66.

17. Marr KA, Laverdiere M, Gugel A, Leisenring W. Antifungal therapy decreases sensitivity of the Aspergillus galactomannan enzyme immunoassay. Clin Infect Dis. 2005;40(12):1762-9.

18. Pinel C, Fricker-Hidalgo H, Lebeau B, Garban F, Hamidfar R, Ambroise-Thomas $\mathrm{P}$, et al. Detection of Circulating Aspergillus fumigatus Galactomannan: Value and Limits of the Platelia Test for Diagnosing Invasive Aspergillosis. J Clin Microbiol. 2003;41(5):2184-6.

19. Barnes PD, Marr KA. Risks, diagnosis and outcomes of invasive fungal infections in haematopoietic stem cell transplant recipients. Brit J Haematol. 2007;139(4):519-31.

20. Parody R, Martino R, Sanchez F, Subira M, Hidalgo A, Sierra J. Predicting survival in adults with invasive aspergillosis during therapy for hematological malignancies or after hematopoietic stem cell transplanation: single-center analysis and validation of the Seattle, French, and Strasbourg prognostic indexes. Am J Hematol. 2009;84(9):571-8.

21. Fukuda T, Boeckh M, Carter RA, Sandmaier BM, Maris MB, Maloney DG, et al. Risks and outcomes of invasive fungal infections in recipients of allogeneic hematopoietic stem cell transplants after nonmyeloablative conditioning. Blood. 2003;102(3):827-33.

22. Bowyer P, Moore CB, Rautemaa R, Denning DW, Richardson MD. Azole antifungal resistance today: focus on Aspergillus. Curr Infect Dis Rep. 2011;13(6):485-91.

23. Denning D, Radford SA, Oakley KL. Correlation between invitro susceptibility testing to itraconazole and in vivo outcome of Aspergillus fumigatus infection. J Antimicrobial Chem. 1997;40 (3):401-14.

24. Van der Linden JW, Snelders E, Kampinga GA, Rijnders BJ, Mattsson E, Debets-Ossenkopp YJ, et al. Clinical implications of azole resistance in Aspergillus fumigatus. The Netherlands, 20072009. Emerg Infect Dis. 2011;17(10):1846-54.

25. Snelders E, Van der Lee HA, Kuijpers J, Rijs AJ, Varga J, Samson RA. Emergence of azole resistance in Aspergillus fumigatus and spread of a single resistance mechanism. PLoS Med. 2008;5(11):e219.

26. Alcazar-FuoliL,MelladoE.Currentstatus ofantifungalresistanceand its impact on clinical practice. Br J Haematol.2014;166(11):471-484. doi: 10.1111/bjh.12896. 
27. Nucci M. Emerging moulds: Fusarium, Scedosporium and zygomycetes in transplant recipients. Curr Opin Infect Dis. 2003;16 (6):607-12.

28. Johnson EM, Szekely A, Warnock DW. In vitro activity of voriconazole, itraconazole and amphotericin B against filamentous fungi. J Antimicrobial Chem. 1998;42(6):741-45.

29. Lalitha P, Shapiro BL, Srinivasan S, Prajna NV, Archarya NR, Fothergill AW, et al. Antimicrobial Susceptibility of Fusarium, Aspergillus, and other filamentous fungi isolated from keratitis. Arch Ophthalmol. 2007;125(6):789-93.

30. Santhanam J, Teong AK, Amran F. Susceptibility testing of clinical mould isolates in Malaysia with the Etest and M38-A methods. J Med Sci. 2008;8(1):94-7.

31. Guitard J, Degulys A, Buot G, Aline-Fardin A, Dannaoui E, Rio $\mathrm{B}$, et al. Acremonium sclerotigenum-Acremonium egyptiacum:a multi-resistant fungal pathogen complicating the course of aplastic anaemia. Clin Microbiol Infect. 2014;20(1):30-32.

32. Neofytos D, Horn D, Anaissie E, Steinbach W, Olyaei A, Fishman $\mathrm{J}$, et al. Epidemiology and outcome of invasive fungal infection in adult hematopietic stem cell transplant recipients: analysis of multicenter prospective antifungal therapy (PATH) alliance. Clin Infect Dis. 2009;48(3):265-73.
33. Clark TA, Slavinski SA, Morgan J, Lott T, Arthington-Skaggs BA, Brandt ME, et al. Epidemiologic and molecular characterization of an outbreak of Candida parapsilosis bloodstream infections in a community hospital. J Clin Microbiol. 2004;42(10):4468-72.

34. Girmenia C, Martino P, De BF, Gentile G, Boccanera M, Monaco M, et al. Rising incidence of Candida parapsilosis fungemia in patients with hematologic malignancies: clinical aspects, predisposing factors, and differential pathogenicity of the causative strains. Clin Infect Dis. 1996;23(3):506-14.

35. Levy I, Rubin LG, Vasishtha S, Tucci V, Sood SK. Emergence of Candida parapsilosis as the predominant species causing candidemia in children. Clin Infect Dis. 1998;26(5):1086-88.

36. Bergamasco MD, Garnica M, Colombo AL, Nucci M. Epidemiology of candidemia in patients with hematologic maligancies and solid tumours in Brazil. Mycoses. 2012;56(3):256-63.

37. de With K, Steib-Bauert MS, Knoth H, Dorje F, Strehl E, Rothe U, et al. Hospital use of systemic antifungal drugs. BMC Clin Pharmacol. 2005; 5:1. doi: 10.1186/1472-6904-5-1.

38. Oberoi JK, Wattal C, Goel N, Raveendran R, Datta S, Prasad K. Non-albicans Candida species in blood stream infections in a tertiary care hospital at New Delhi, India. Indian J Med Res. 2012;136(6):997-1003. 